\title{
Structural Ordering and Composition of Warner Mountains Obsidian and its Microlites
}

\author{
Ellis Kennedy ${ }^{1}$, Bengisu Sari ${ }^{1}$ and Mary $\operatorname{Scott}^{2}$ \\ ${ }^{1}$ University of California, Berkeley, Berkeley, California, United States, ${ }^{2} \mathrm{UC}$ Berkeley, Berkeley, \\ California, United States
}

Obsidian is an amorphous mineraloid that forms when highly silicic viscous lava cools rapidly before a crystal structure can develop. The composition and structure of obsidian is highly dependent on the magma from which it formed and the kinetics governing its solidification. Overtime, obsidian devitrifies, becoming more atomically ordered [1]. The amorphous matrix is comprised mainly of $\mathrm{SiO}_{2}$ (typically over 70\%) and oxides of $\mathrm{Al}, \mathrm{Ti}, \mathrm{Fe}, \mathrm{Ca}, \mathrm{Na}, \mathrm{K}, \mathrm{Mg}$, and $\mathrm{Mn}$. A host of other elements are also typically present in trace quantities [2]. Characterization of the composition of obsidian and its microlites provides information on its place of origin and the composition of its parent magma. Structural characterization of the amorphous matrix provides information on its degree of disorder and the uniformity of its short- and medium- range order. Obsidian is brittle and exhibits conchoidal fracture when struck with force [3]. Sharp, thin edges are achievable when shards are cleaved from bulk samples. For the same reason that obsidian was used for tool making by ancient civilizations, it can easily be prepared for analysis in the TEM. Millimeter-scale samples were created using flint knapping techniques and adhered to standard TEM grids with the distal edge in the center.

The obsidian analyzed in this study was sourced from the Warner Mountains in Northern California, along the northwestern margin of the Basin and Range province. They formed during the mid-Miocene [4]. Bulk samples of both red and black obsidian were analyzed.

Using a TitanX, energy-dispersive x-ray spectroscopy (EDS) maps and diffraction data were collected from regions of the obsidian with homogeneous composition and from regions with nano- and micronscale microlites. Figure 1 shows EDS maps collected from a region with microlites. Within the amorphous matrix, microlites were identified based on their major chemical elements: iron oxides (Fe-Cu-Mn-O), $\mathrm{Ca}-\mathrm{Mg}-\mathrm{Fe}-\mathrm{Cu}-\mathrm{Mn}, \mathrm{Na}-\mathrm{S}-\mathrm{Cl}$, and titanium oxide (Ti-O). The host matrix was confirmed to be amorphous using diffraction techniques and was found to be predominantly Si-Al-O with varying amounts of $\mathrm{Na}, \mathrm{K}$, and other elements. $\mathrm{Al}, \mathrm{Na}$, and $\mathrm{K}$ depletion is often observed near microlites containing $\mathrm{Ca}$ and $\mathrm{Mg}$. This is indicative of elemental diffusion after the obsidian formed.

Collection of scanning nanodiffraction data necessitates exceptionally thin sample regions, so it was only collected from the very edges and from regions where the compression band structure of the obsidian created larger sheaths of uniform thickness. The compressional cracking ripples are broken if samples are polished or dimpled, adding further reason to take advantage of the natural fracture properties of obsidian. 4D-scanning transmission electron microscopy maps were taken from regions with and without visible microlites. Using a Tecnai equipped with an electron energy-loss spectrometer, these regions were determined to have a thickness ranging from 50 to $120 \mathrm{~nm}$.

The data was processed using the py4DSTEM analysis toolbox and custom Matlab scripting $[5,6]$. Fluctuation electron microscopy (FEM) is a scanning nanodiffraction technique that is uniquely sensitive to medium-range ordering on the length-scale of the probe diameter [7]. An FEM approach was applied to the uniformly thin edges to determine the average bond length and to get an idea of the homogeneity of 
medium range ordering over a region of a few microns. Data sets consisting of over 2,000 images were acquired for statistical analysis. With a probe diameter of $2.5 \mathrm{~nm}^{-1}$, structural information was obtained on the $2-5 \mathrm{~nm}$ length scale from the obsidian samples. Variance in mean radially integrated intensity was observed to increase around a scattering vector of $2.67 \mathrm{~nm}^{-1}$, corresponding to an average bond length of $3.75 \AA$. These values were consistent across the thin regions. Figure 2 shows FEM and parallel beam diffraction from a thin region of compressional bands along the edge of a sample with no microlites.

Short-range order was evaluated using the py4DSTEM radial distribution function (RDF) notebook and eRDF Analyser [5,8]. Average pixel intensity maps and scanning nanodiffraction patterns were used to identify regions near and on microlites for RDF analysis. The obsidian matrix provides amorphous structural information and the microlites provide crystalline information. Attention is given to the microlite-amorphous matrix interface. The diffraction information was combined with the EDS chemical composition maps to correlate changes in obsidian structure with the presence of microlites.

This work provides on example of diffraction techniques applied to a nonuniform earth sample for structural characterization. This technique allows for understanding of the structure-composition relationship of obsidian and has the potential to provide information on the degree of disorder in obsidian, the effect of elemental diffusion near microlites on the structure of the $\mathrm{Si}-\mathrm{Al}-\mathrm{O}$ matrix, and the magmatic environment from which the obsidian originated.

Work at the Molecular Foundry was supported by the Office of Science, Office of Basic Energy Sciences, of the U.S. Department of Energy under Contract No. DE-AC02-05CH11231.

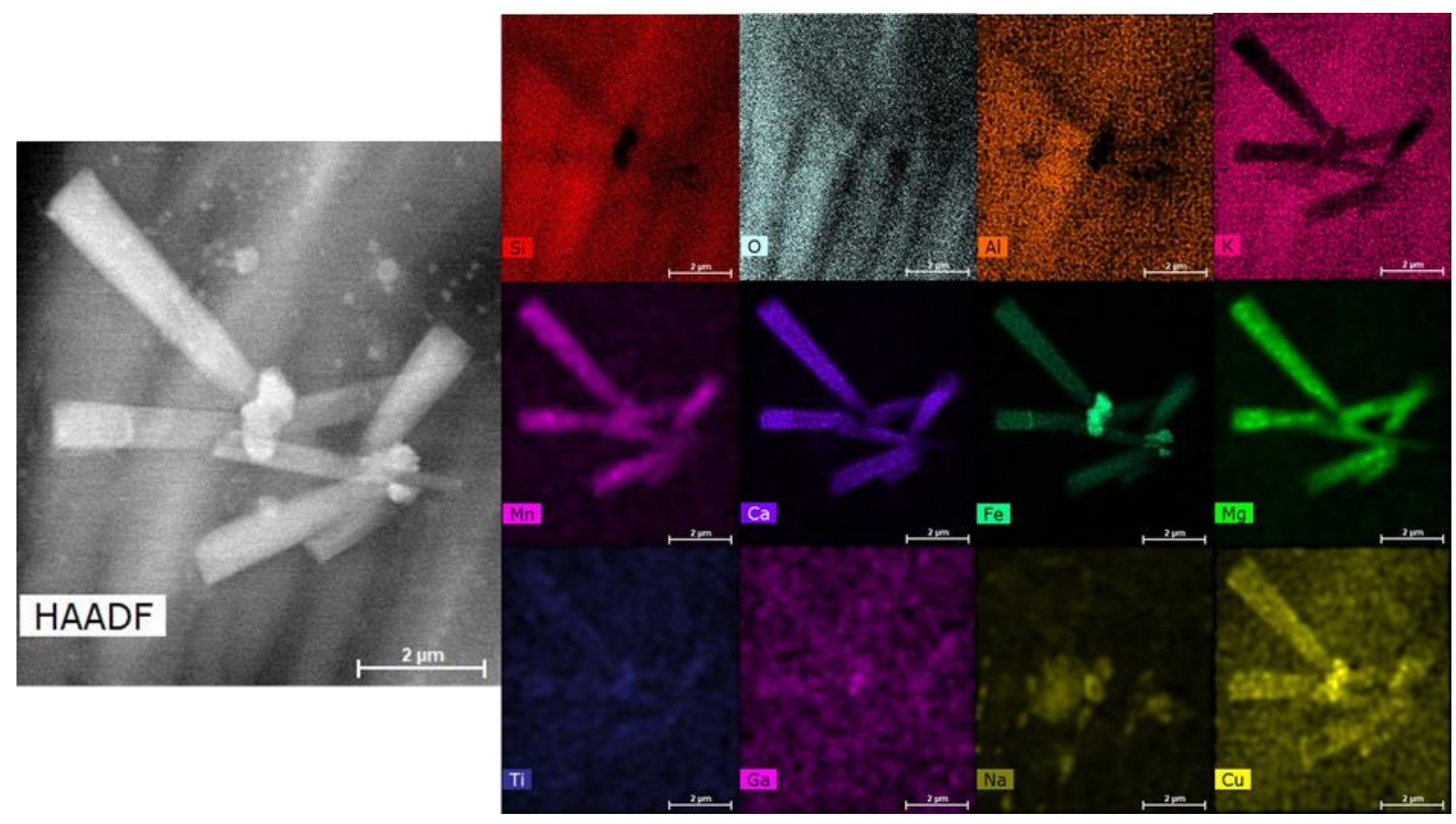

Figure 1. EDS map taken of Fe-Mn-Mg-Ca microlites. K-depletion is observed where the microlites are embedded in the amorphous matrix. Similar Fe-based and microlites were observed throughout the samples. $\mathrm{TiO} 2$ and plagioclase crystals were also observed. 

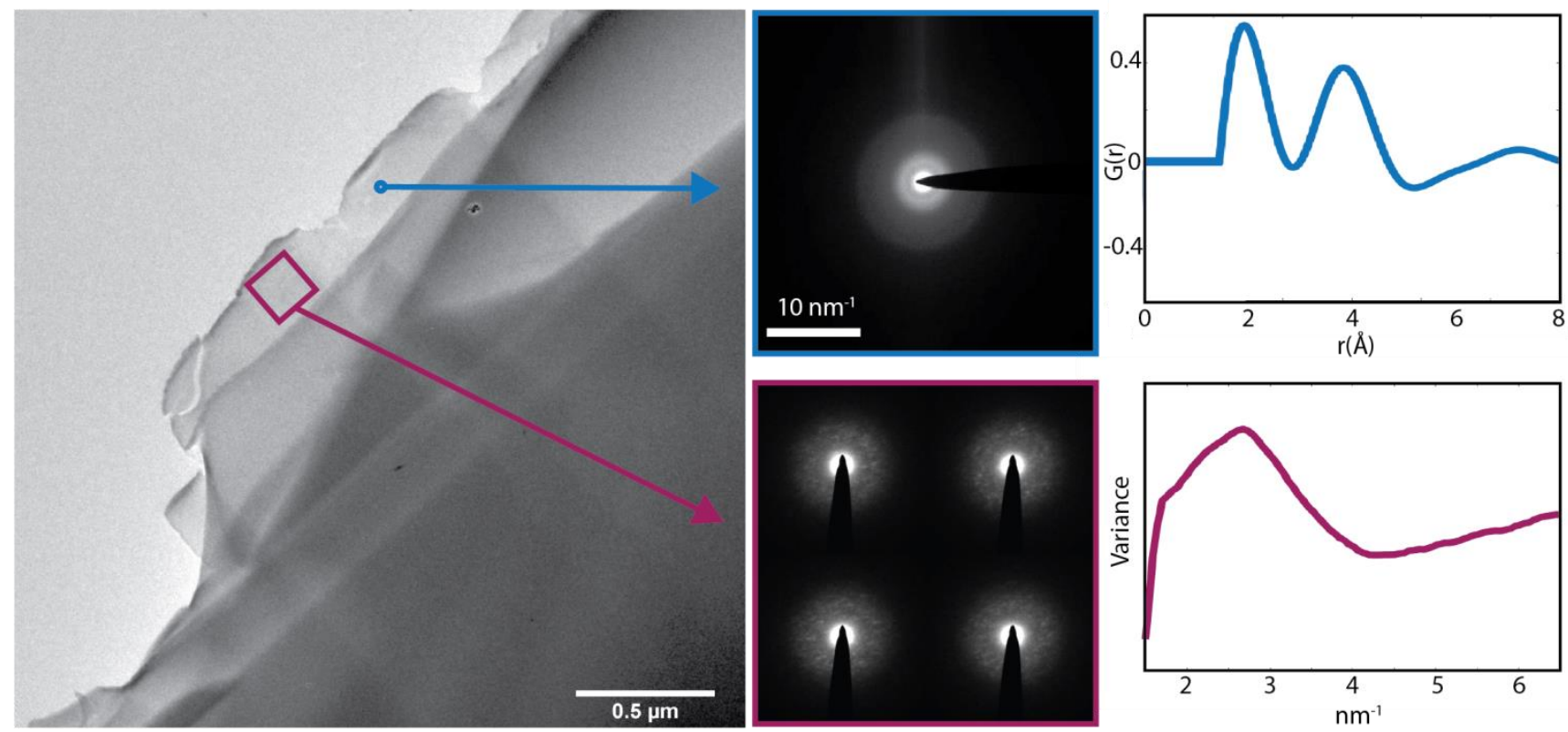

Figure 2. A thin edge of obsidian. Parallel beam diffraction and scanning nanodiffraction data were taken from a region approximately 50-nm thick. Over 2,000 scanning nanodifraction images were collected; the four shown are a representative sample of the data. From the diffraction data, RDF and variance calculations were performed.

\section{References}

[1] Jerram, D. and Petford, N., 2011. The Field Description of Igneous Rocks, $2^{\text {nd }}$ ed., pp. 71-82.

[2] Sano, K. and Toramaru, A. Journal of Volcanology and Geothermal Research, 341, pp. 158-171.

[3] Miskuf, J. et al., 2017. Materials Science Forum, 891, pp. 504-508.

[4] Putirka, K. and Platt, B., 2012, Geosphere, 8, pp. 1274-1285.

[5] Savitsky, B., et al., 2020, arXiv:2003.09523.

[6] Kennedy, E., et al., 2020. Applied Physics Letters, 117.

[7] Voyles, P.M. and Muller, D.A., 2002. Ultramicroscopy, 93(2), pp.147-159.

[8] Shanmugam, J., et al., 2017, SoftwareX, 2017, 6, pp. 185-192. 